\title{
Molecular Dynamics Simulation of Solvent Reorganization in Ion Transfer Reactions near a Smooth and Corrugated Surface
}

\author{
Christoph Hartnig ${ }^{\dagger}$ and Marc T. M. Koper* \\ Laboratory of Inorganic Chemistry and Catalysis, Schuit Institute of Catalysis, \\ Eindhoven University of Technology, 5600 MB Eindhoven, The Netherlands
}

Received: December 17, 2003

\begin{abstract}
We study the influence of different metal-water potentials on the energetics of ion transfer reactions at metal electrodes by extensive molecular dynamics simulations. The (slope of the) barrier for both ion and atom adsorption is found to be higher for a corrugated metal-water potential compared to a smooth metalwater potential, due to the more rigid water structure caused by the former potential. Interestingly, between 4 and $6 \AA$ from the surface, the free energy profiles are the same for both ion and atom, suggesting that the displacement of the water from the surface makes the largest contribution to the free energy of adsorption. Although the parameters for solvent reorganization related to the ion/atom transfer depend critically on the details of the metal-water potential, this is much less so for the solvent reorganization due to electron transfer. The small differences observed in solvent reorganization energy and charge transfer are due to the different simulation boxes used for the two different potentials, rather than to intrinsically different energetics. Therefore, solvent reorganization related to electron transfer is primarily governed by long-range electrostatic effects, whereas solvent reorganization related to ion transfer is primarily governed by much shorter-range solvent structural effects existing at the electrode-electrolyte interface.
\end{abstract}

Electrode processes often feature reactions in which a species has to be transferred across the double layer, either from the adsorbed state into solution or vice versa. These processes are controlled not only by the interaction with the (metal) electrode but also to a considerable extent by the interaction with the solvent/electrolyte, especially when charged species are involved. A unified model for such ion transfer reactions was put forward a few years ago by Schmickler. ${ }^{1-4}$ In Schmickler's model, two types of solvent reorganization that accommodate ion transfer are incorporated: solvent reorganization due to the transfer of the ion through the electric double layer, ${ }^{5,6}$ by which the ion has to partially shed off or buildup (part of) its solvation shell, and solvent reorganization related to the electron transfer between the ion and the electrode. The latter is the kind of solvent reorganization considered in the Marcus theory of electron-transfer reactions. ${ }^{7}$ Schmickler's model treats both types of solvent reorganization on a phenomenological or parametrized level, allowing for a calculation of the full potential energy surface of ion transfer reactions as a function of the generalized solvent coordinate known from the theory of electron transfer reactions and the distance of the species from the electrode. This model has been very useful in providing a detailed energetic picture of ion transfer reactions, especially when neutral species and/or more than two transferring electrons are involved, ${ }^{4}$ and has pointed out some inconsistencies in previous models and ideas about such electrode reactions.

We have recently presented extensive molecular dynamics (MD) simulations of a fully molecular version of Schmickler's model, ${ }^{8}$ in which the interaction of the redox species with the

\footnotetext{
* Corresponding author. E-mail: mtmkoper@tue.nl.

† Present address: Forschungszentrum Jülich, Institute for Materials and Processes in Energy Systems (IWV-3), D-52425, Jülich, Germany. Email: c.hartnig@fz-juelich.de.
}

solvent is treated without any phenomenological assumptions other than the model potentials chosen for the pairwise interactions. As in Schmickler's model, the electron transfer between the redox species and the metal electrode was incorporated by the Anderson-Newns Hamiltonian, ${ }^{9}$ as first applied to MD simulations by Straus et al.. ${ }^{10}$ One of the main assumptions of our previous work was related to the type of metal-water interaction that was included in the simulation. This interaction essentially implied a smooth surface with metallic properties, with no preferential interaction of the water molecule for a particular coordination to the surface. As water molecules tend to adsorb preferentially to one single metal surface atom, ${ }^{11}$ a more realistic model potential should take into account this corrugation of the water-metal interaction. Indeed, water at smooth model surfaces tends to be more mobile compared to water at corrugated surfaces. ${ }^{12-14}$ In this Article, we will present results of MD simulations of ion transfer reactions at surfaces with smooth and corrugated metal-water potentials. Not only is this an extension as well as an improvement compared to our previous simulations, it also allows us to assess the importance of the details of the metalwater interactions on features such as the ion transfer barrier, the extent of electron transfer, the distance dependence of the solvent reorganization parameters, etc.

The details of the simulation procedures have been described elsewhere $^{8}$ and will not be reiterated here. Briefly, for the smooth surface the simulation box (with a square base of 24.82 $\times 24.82 \AA$ ) contained 511 water molecules, interacting with each other through the SPC/E model potential, 1 redox species, and a surface. The ion-water potential was taken from Lee and Rasaiah, ${ }^{15}$ and the radius of the ion was chosen such that it typically represents a chloride $\mathrm{Cl}^{-}$or chlorine $\mathrm{Cl}^{0}$ species. Two different kinds of metal-water potentials were employed. 


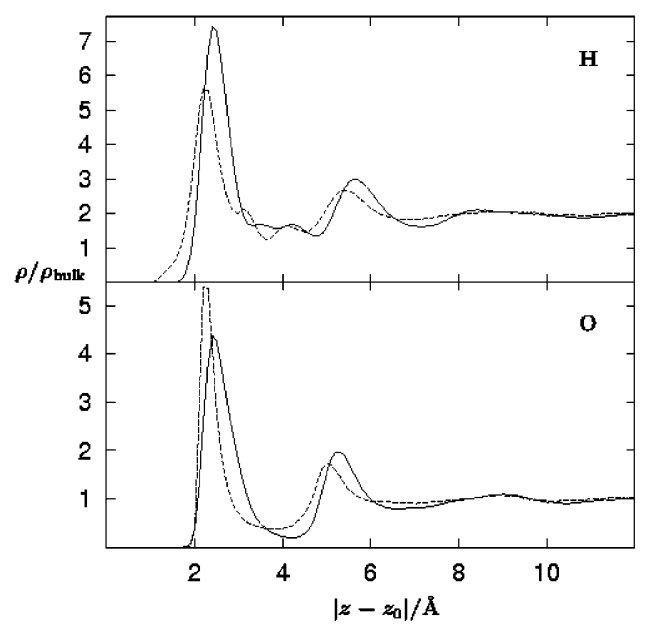

Figure 1. Hydrogen and oxygen distribution functions for the corrugated metal-water potential (solid line) and the smooth metalwater potential (dashed line). For the corrugated metal-water potential $\mathrm{z}_{0}$ was defined at the center of the platinum surface atoms. ${ }^{16}$

The smooth model potential considered in our previous work is modeled by

$$
V(z)=4 \epsilon\left[\left(\frac{\sigma}{z-z_{0}}\right)^{12}-\left(\frac{\sigma}{z-z_{0}}\right)^{6}\right]
$$

where $z_{0}$ denotes the position of the wall and $z$ is the actual position of the atom under consideration. The values for $\epsilon$ and $\sigma(\epsilon=30.1 \mathrm{~kJ} / \mathrm{mol}, \sigma=2.0 \AA)$ were parametrized in such a way that the density distribution perpendicular to the surface that mimics the well-known density distribution in systems with atomically resolved metal surfaces. For the corrugated surface, the simulation box was $37.294 \AA$ by $19.22299 \AA$ containing $539 \mathrm{SPC} / \mathrm{E}$ water molecules and one redox species. This rectangular shape was chosen to fit a hexagonal 111 surface, as employed by Straus et al. ${ }^{10}$ The corrugated model potential was the one suggested by Berkowitz et al. ${ }^{16}$ for the water/Pt(111) interface. The expressions are rather lengthy and can be found in the original paper. To prevent the water molecules from evaporating, a slightly repulsive potential at the water-vacuum interface was applied such that the experimental density of 1.0 $\mathrm{g} / \mathrm{cm}^{3}$ is achieved in the center of the water slab. Long-range interactions were treated by the Ewald summation with correction for 2D periodic systems. ${ }^{17}$ Figure 1 compares the $z$ dependent $\mathrm{O}$ and $\mathrm{H}$ distribution functions for the two potentials.

During the simulation, a two-dimensional phase space was sampled, spanned by the distance of the redox species from the electrode surface and the generalized solvent coordinate $\Delta E$. The definition and calculation of this latter quantity has been described in detail elsewhere. ${ }^{10,18,19}$ To sample improbable values of $\Delta E$, a two-dimensional umbrella sampling procedure was adopted. ${ }^{8,18}$ (Typically, the simulation time for the equilibrium simulation, i.e., without umbrella potential, was 300$400 \mathrm{ps}$, whereas data sampling with the umbrella potential was done for 150 ps per umbrella window.) The amount of charge transfer between the metal electronic levels and the solute's orbital is obtained from the following formula:

$$
n(\Delta E)=\frac{1}{2}+\frac{1}{\pi} \tan ^{-1}\left(\frac{\epsilon_{\mathrm{F}}-\epsilon_{\mathrm{a}}-\Delta E}{\Delta(z)}\right)
$$

where $n$ is the occupation number of the acceptor orbital, $\epsilon_{\mathrm{F}}$ is the Fermi level of the metal (taken $-5.3 \mathrm{eV}$ as representative for platinum), $\epsilon_{\mathrm{a}}$ is the vacuum energy level of the acceptor's

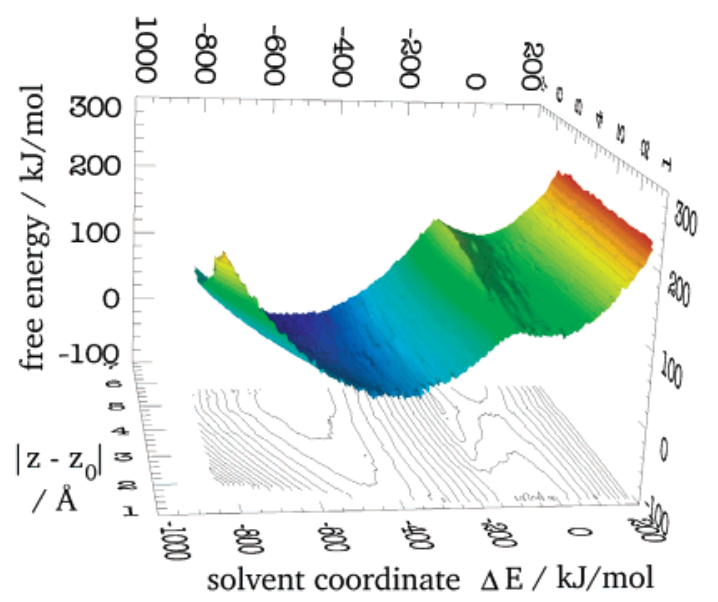

225
200
175
150
125
100
75
50
25
25

(a)

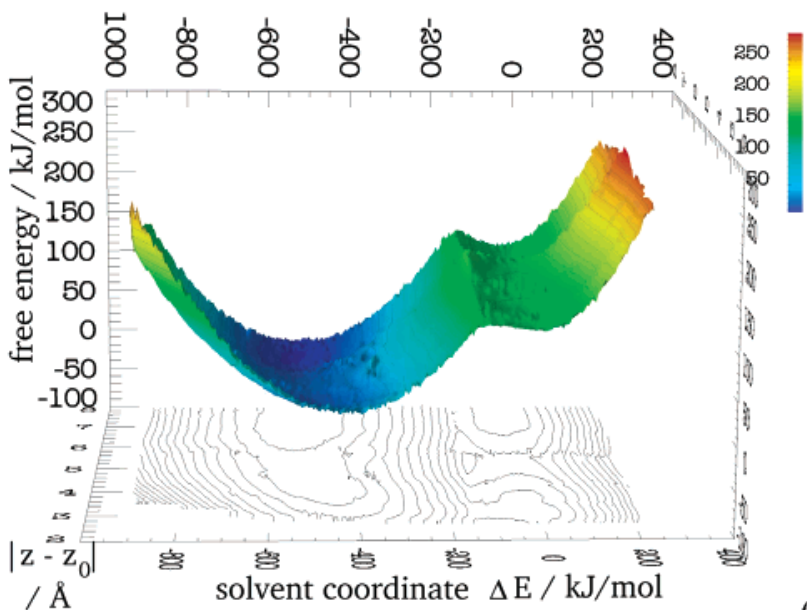

(b)

Figure 2. Free energy surfaces for the $\mathrm{Cl}^{-} / \mathrm{Cl}$ couple for (a) the smooth metal-water potential and (b) the corrugated metal-water potential.

electron orbital (taken $3.614 \mathrm{eV}$ as representative for chlorine), and

$$
\Delta(z)=\Delta_{0} \exp (-b z)
$$

This latter quantity describes the strength of the quantummechanical interaction in terms of the broadening of the solute's orbital energy. The exponential distance dependence is suggested by ab initio quantum-chemical calculations. ${ }^{20}$ The values taken for $\Delta_{0}$ and $b$ were $2 \mathrm{eV}$ and $1 \AA^{-1}$, respectively. We note that the present simulation does not incorporate the image force between the ion and the metal surface, and hence all potential energy surfaces correspond to solvent potentials of mean force. ${ }^{5}$ In any case, the image force is essentially an additive term (as long as charge transfer is not too large) and could therefore easily be incorporated.

Figure 2 shows the potential energy surfaces obtained for the $\mathrm{Cl}^{-} / \mathrm{Cl}$ redox couple for the smooth interaction potential (Figure 2a) and the corrugated interaction potential (Figure 2b). The reaction paths for chloride and chlorine can be clearly discerned. As expected, the energy for $\mathrm{Cl}$ is considerably higher, making a direct electron-transfer event $\mathrm{Cl}^{-} \rightarrow \mathrm{Cl}+\mathrm{e}^{-}$a very unlikely process. Also note that, close to the electrode surface, the barrier between the $\mathrm{Cl}^{-}$and $\mathrm{Cl}$ channels disappears as a result of the strong electronic interaction (large value of the $\Delta(z)$ parameter).

The reaction paths for chloride and chlorine adsorption as a function of the distance to the surface are compared in Figure 3. It is observed that the slope for ion adsorption in case of a 


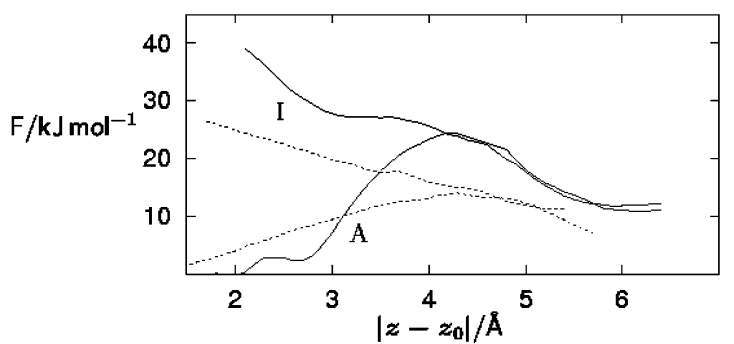

Figure 3. Free energy profiles for the minima in Figure 2 as a function of distance from the surface. Solid lines are the curves for the atom ("A") and ion ("I") with the corrugated metal-water potential; dashed lines are the curves for the atom and ion with the smooth metal-water potential.

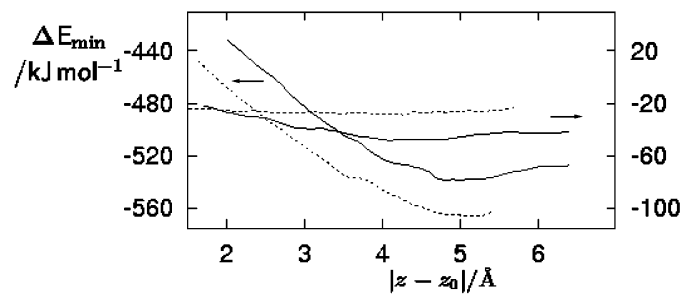

Figure 4. Values of the solvent coordinate in the minima of Figure 2 as a function of distance from the surface. Solid lines are the curves for the atom ("A") and ion ("I") with the corrugated metal-water potential; dashed lines are the curves for the atom and ion with the smooth metal-water potential.

corrugated surface is higher than for a smooth surface, and the barrier for atom adsorption is higher for a corrugated surface compared to a smooth surface. As mentioned in our previous paper, this is not surprising, as the corrugated surface has a more rigid interfacial water structure, which is more difficult to displace. The barrier for atom adsorption is due to a finite free energy penalty related to making a "hole" between the atom and the surface in which it is difficult to place a water molecule. Interestingly, the shapes of the free energy curves of the atom and ion at distances between 4 and $6 \AA$ from the surface are almost identical. This strongly suggests that at these distances, the main contribution to the free energy barrier stems from the displacement of the surface-bonded water, and not from the breaking of the solvation shell. Between 3 and $3.5 \AA$, there is a flat region in the free energy curve for the ion with the corrugated metal-water potential, which coincides with a low density of water (oxygen) in the distribution function (see Figure 1), implying that the ion can move relatively freely. For shorter distances, the solvent potential mean of force for the ion increases again, due to the need to break down the solvation shell, whereas the atom becomes attracted to the surface because it can more easily strip off its solvation shell.

Figure 4 depicts the minimum of the solvent coordinate $\Delta E$ as a function of the distance from the surface for both species and for both metal-water potentials. This quantity represents the electrostatic part of the solvent-solute interaction, and it is observed to decrease as the ion approaches the surface (for the atom, the distance dependence is very minor). The absolute values of this quantity for the ion are slightly different (by ca. $30 \mathrm{~kJ} / \mathrm{mol}$ ) for the two different metal-water potentials, which is mainly due to the different simulation box sizes and setup. However, the slopes of the curves are essentially indistinguishable, which implies that the long-range electrostatic forces between the ion and the solvent for the two different models are very similar. Figure 5 shows the solvent reorganization energy $\lambda$ as a function of the distance for both species and for both metal-water interactions. This parameter was estimated

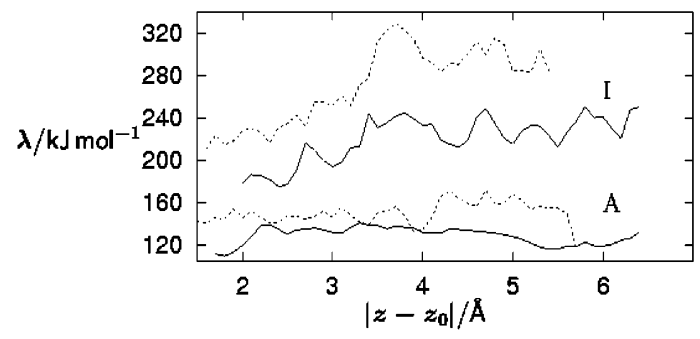

Figure 5. Solvent reorganization energies as a function of distance from the surface. Solid lines are the curves for the atom and ion with the corrugated metal-water potential; dashed lines are the curves for the atom and ion with the smooth metal-water potential.

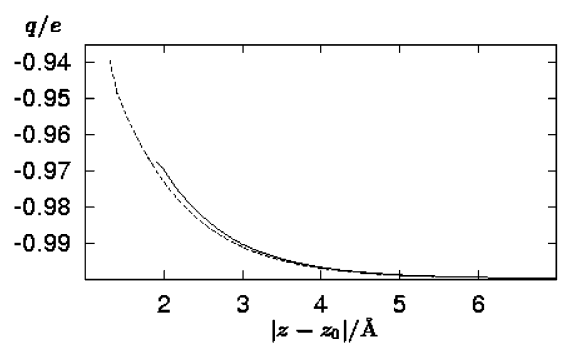

Figure 6. Charge on the chloride species as a function of the distance from the surfaces. The solid line is for the corrugated metal-water potential; the dashed line for the smooth metal-water potential.

at the minimum of each channel from the curvature of the potential energy surface along the solvent coordinate. ${ }^{10,17}$ Here, a similar behavior as for the minimum of the solvent coordinate is observed. The absolute value of the solvent reorganization energy for the corrugated metal-water potential is about 50$60 \mathrm{~kJ} / \mathrm{mol}$ higher than for the smooth potential, but the dependence of $\lambda$ on the distance is essentially identical. Again, the quantitative differences are ascribed to the differences in the computational setup. In agreement with our earlier results, it is found that for the ion $\lambda$ decreases as the surface is approached, whereas for the atom it is almost constant. This is attributed to the role of long-range solute-solvent electrostatic interactions: these are strongly distance dependent when the solute is an ion, but almost absent when the solute is an atom, for which only short-range interactions are important.

Finally, Figure 6 plots the charge on the chloride species as a function of the distance from the surface for both metalwater potentials. This charge was calculated from eq 2. A small charge transfer is observed as the electrode is approached. The small difference between the two simulations is again ascribed to the different simulation boxes: the different electrostatics will lead to different electrostatic shifts of the orbital energy level and hence to different degrees of electron transfer.

In conclusion, we have studied the influence of different metal-water potentials on the energetics of ion transfer reactions at metal electrodes by extensive molecular dynamics simulations. We have found that the (slope of the) barrier for both ion and atom adsorption is higher for a corrugated metal-water potential than for a smooth metal-water potential, due to the more rigid water structure caused by the former. In fact, between 4 and $6 \AA$ from the surface, the free energy profiles are the same for both ion and atom, suggesting that the displacement of the water from the surface makes the largest contribution. Although the free energy parameters of solvent reorganization related to the ion/atom transfer depend critically on the details of the metal-water potential, this is much less so for the solvent reorganization due to electron transfer. The small differences observed in solvent reorganization and charge transfer are due to the different simulation boxes used for the two different 
potentials, rather than to intrinsically different energetics. This leads to the conclusion that solvent reorganization related to electron transfer is primarily governed by long-range electrostatic effects, whereas solvent reorganization related to ion transfer is primarily governed by the much shorter-range solvent structural effects existing at the electrode-electrolyte interface.

Acknowledgment. This research was supported by The Netherlands Organization for Scientific Research (NWO) and by a generous grant of supercomputer facilities by The Netherlands National Computing Facilities Foundation (NCF).

\section{References and Notes}

(1) Schmickler, W. Chem. Phys. Lett. 1995, 237, 152.

(2) Schmickler, W. Electrochim. Acta 1996, 41, 2329.

(3) Koper, M. T. M.; Schmickler, W. In Electrocatalysis; Lipkowski, J., Ross, P. N., Eds.; Wiley-VCH: New York, 1998; p.291. 83.

(4) Koper, M. T. M.; Schmickler, W. J. Electroanal. Chem. 1998, 450,
(5) Spohr, E. Chem. Phys. Lett. 1990, 141, 87.

(6) Pecina, O.; Schmickler, W.; Spohr, E. J. Electroanal. Chem. 1995, 394,29

(7) (a) Marcus, R. A. J. Chem. Phys. 1956, 24, 966. (b) Marcus, R. A. J. Chem. Phys. 1965, 43, 679. (c) Marcus, R. A. Angew. Chem. Int. Ed. Engl. 1993, 32, 1111

(8) Hartnig, C.; Koper, M. T. M. J. Am. Chem. Soc. 2003, 125, 9840.

(9) Muscat, J. P.; Newns, D. M. Prog. Surf. Sci. 1978, 9, 1.

(10) Straus, J. B.; Calhoun, A.; Voth, G. A. J. Chem. Phys. 1995, 102 529.

(11) Thiel, P. A.; Madey, T. E. Surf. Sci. Rep. 1987, 7, 211.

(12) Schonnenschein, R.; Heinzinger, K. Chem. Phys. Lett. 1983, 102, 550

(13) Wallqvist, A. Chem. Phys. Lett. 1990, 165, 437.

(14) Lee, S. H.; Rossky, P. J. Chem. Phys. 1994, 100, 3334

(15) Lee, S. H.; Rasaiah, J. C. J. Phys. Chem. 1996, 100, 1420

(16) Raghavan, K.; Foster, K.; Motakabbir, K.; Berkowitz, M. J. Chem. Phys. 1991, 94, 2110.

(17) Yeh, I. C.; Berkowitz, M. J. Chem. Phys. 1999, 111, 3155.

(18) Hartnig, C.; Koper, M. T. M. J. Chem. Phys. 2001, 115, 8540.

(19) Warshel, A. J. Phys. Chem. 1982, 86, 2218.

(20) Koper, M. T. M.; van Santen, R. A. Surf. Sci. 1998, 422, 118. 